\title{
Intereye and intraeye asymmetry analysis of retinal microvascular and neural structure parameters for diagnosis of primary open-angle glaucoma
}

\author{
Huan Xu ${ }^{1,2,3} \cdot$ Yuan Zong ${ }^{1,2,3} \cdot$ Ruyi Zhai ${ }^{1,2,3} \cdot$ Xiangmei Kong $^{1,2,3} \cdot$ Chunhui Jiang ${ }^{1,2,3} \cdot$ Xinghuai Sun $^{1,2,3,4}$
}

Received: 17 November 2018 / Revised: 28 March 2019 / Accepted: 6 April 2019 / Published online: 3 May 2019

(c) The Royal College of Ophthalmologists 2019

\begin{abstract}
Purpose To investigate the diagnostic values of intereye or intraeye asymmetry of retinal perfused vessel density and neural structure parameters for detection of glaucoma.

Methods In total, 152 healthy subjects and 72 bilateral primary open-angle glaucoma (POAG) patients were enrolled. Total POAG group contains all glaucoma patients. Early to moderate POAG group contains patients whose binocular mean defect values were larger than $-12 \mathrm{~dB}$. The retinal perfused vessel densities were acquired using optic coherence tomography angiography. The neural structure parameters include RNFL, GCC thickness and its derivative indices like focal loss volume percentage (FLV\%) and global loss volume percentage (GLV\%). Intereye asymmetry equaled to the absolute difference of parameters between paired eyes. Intraeye asymmetry was defined as absolute difference between the inferior and superior hemisphere values from one random selected eye. The areas under the receiver operating characteristic curves (AUROCs) were calculated to evaluate diagnostic ability.

Results From pairwise comparison analysis of ROC curves, the intereye asymmetric parameters with the largest diagnostic accuracy were FLV and GLV\% (AUROC =0.944), which were significantly superior to the intereye asymmetry of perfused vessel density in peripapillary area and parafoveal area $(P<0.05)$. Particularly, the intereye asymmetry of FLV\% $(A U R O C=0.926)$ and GLV\% (AUROC $=0.950)$ showed excellent diagnostic precision for detecting early to moderate glaucoma patients. However, the intraeye asymmetry of microvascular parameters and neural structure parameters showed fair diagnostic ability for identifying POAG patients.

Conclusions The intereye asymmetry of neural structure parameters, particularly the FLV\% and GLV\%, outperformed the microvascular parameters for identifying POAG patients.
\end{abstract}

These authors contributed equally: Huan $\mathrm{Xu}$, Yuan Zong

Supplementary information The online version of this article (https:// doi.org/10.1038/s41433-019-0451-x) contains supplementary material, which is available to authorized users.

Xiangmei Kong

kongxm95@163.com

$\triangle$ Chunhui Jiang

chhjiang70@163.com

1 Department of Ophthalmology and Visual Science, Eye, Ear, Nose, and Throat Hospital, Shanghai Medical College, Fudan University, Shanghai, China

\section{Introduction}

Symmetry is an important feature of paired organs. Primary open-angle glaucoma (POAG) is a chronic optic neuropathy characterized by progressive loss of retinal ganglion cell and visual field defects [1]. It was reported that asymmetry was an important sign of early glaucomatous damage [2]. The assessment of differences relative to normal subjects provides the possibility of evaluating asymmetric features

2 Key Laboratory of Myopia, Ministry of Health (Fudan University), Shanghai, China

3 Shanghai Key Laboratory of Visual Impairment and Restoration (Fudan University), Shanghai, China

4 State Key Laboratory of Medical Neurobiology, Institutes of Brain Science, Fudan University, Shanghai, China 
for the detection of changes caused by the glaucoma disease.

Previous reports have demonstrated that the intereye differences in intraocular pressure (IOP) [3], optic disc parameters [4-6], central corneal thickness [7, 8], retinal nerve fiber layer (RNFL) thickness $[9,10]$, macular thickness [9, 11], and visual field defects [12] are all associated with glaucoma disease progression. Additionally, intraeye asymmetry of neural structure and functional parameters between the superior and inferior hemisphere often occurs with glaucomatous changes. Several studies have reported that parameters from intraeye asymmetry analysis of macular or RNFL hemifield thickness could be useful indicators of early-stage POAG [8, 13, 14]. Furthermore, hemifield asymmetry in visual sensitivity using the Glaucoma Hemifield Test (GHT) with standard automated perimetry has been demonstrated to be a good diagnostic parameter for detection of early glaucoma $[15,16]$.

Many studies have provided evidence that abnormal changes of ocular blood flow and vascular autoregulation ability have an important effect on the etiology and progression of glaucoma. However, little is known about intereye or intraeye asymmetry analysis of ocular circulatory parameters due to the limitations of the technique. Recently, using optic coherence tomography angiography (OCTA), Jia et al. [17] realized the quantification retinal perfused vessel density (PVD) at the capillary level with high repeatability [17-19]. Previous studies indicated that the changes of retinal PVDs at both the peripapillary and parafoveal regions were positively correlated with retinal structure and visual function damage due to POAG [19-21]. Several reports demonstrated that retinal PVDs had significant diagnostic ability for POAG; However, the ability was lower than that of RNFL or macular thickness [22, 23]. Currently, updated software (RTVue-XR Avanti, software version 2.0.5.39; Optovue, Inc.; Meridianville, AL, USA) within the spectral domain system provides multisectoral assessment of retinal PVD, which enables a direct comparison of these values between reciprocal sectors of fellow eyes or between the upper and lower halves from a single eye. Thus, we performed this study to evaluate intereye and intraeye asymmetry in retinal PVDs and neural structure parameters. By calculating the sensitivities and areas under the receiver operating characteristic curves (AUROCs) for the asymmetric parameters, we aimed to identify certain potential indices for the detection of POAG, especially for the early-stage of POAG patients.

\section{Methods}

The conduct of study conformed to the tenets of the Declaration of Helsinki and approved by the Institutional
Review Board of the Eye and ENT Hospital of Fudan University, Shanghai, China (No. 2014043). All subjects provided written informed consent before they participated in this study.

\section{Participants and examinations}

From August 2016 to July 2017, 72 patients diagnosed with bilateral POAG who at the Eye and ENT Hospital of Fudan University were recruited. One-hundred fifty-two age- and gender-matched healthy subjects who hold normal medical checkup report were recruited from community. The thorough ophthalmologic examinations were performed on all subjects, including recording of ocular and systemic disease and family history, the slit-lamp biomicroscopy, detailed gonioscopy, and fundus examination. It also included measurement of best-corrected visual acuity (BCVA), IOP, refractive error, and axial length. Visual field (VF) testing was conducted with a standard automated perimetry (Humphrey field analyzer, Carl Zeiss Meditec SITA with 30-2 full-threshold program, Inc., Dublin, CA).

The inclusion criteria for healthy subjects were as follows: BCVA $>0.8$; a spherical equivalent refractive error between $-8.00 \mathrm{D}$ and $+3.00 \mathrm{D}$; normal range of IOP $(<21$ $\mathrm{mmHg}$ ); no presence of glaucomatous optic neuropathy; absence of retinopathy; exclusion of the cardiovascular disease by thorough medical checkup. We enrolled POAG patients as the following criteria: (1) at least twice reliable VF testing and the presence of glaucomatous visual field defects (VFDs), which were confirmed by two glaucoma specialists according to Hodapp-Parrish-Anderson (H-P-A) criteria; (2) glaucomatous optic neuropathy, which was defined as focal or diffuse loss of the neuroretinal rim, notching of optic disc with or without hemorrhage and RNFL defect consistent with the VF deficits; (3) open anterior chamber angles in both eyes, and (4) existence of central fixation.

Total POAG group contains all of the enrolled bilateral glaucoma patients. Moreover, early to moderate stage of POAG group was defined as patients whose binocular MD values were larger than $-12 \mathrm{~dB}$.

\section{Measurement of RNFL and GCC thicknesses}

The RNFL and GCC thicknesses were acquired with the corresponding protocols embedded in the same OCT system referred above. The RNFL map scanning and measurement of RNFL thickness were conducted in a $3.45-\mathrm{mm}$ diameter annulus centered on the optic disc. The following parameters could be read: average RNFL thickness, the inferior RNFL thickness, and superior RNFL thickness (Fig. S1A, B). Image scanning for GCC thickness covered a $7 \times 7 \mathrm{~mm}$ region centered $1 \mathrm{~mm}$ temporal to the fovea. The 
GCC was defined as the distance between the ILM to the inner plexiform layer. Based on the GCC map, the following indices of GCC thickness were automatically computed by the system: average GCC thickness, average hemisphere GCC thickness (inferior and superior), focal loss volume percentage (FLV\%), and global loss volume percentage (GLV\%) (Fig. S1C, D). The FLV\% is the sum of fractional deviation obtained from the significant focal loss area, which is beyond the fifth percentile of normal range. The GLV\% is the sum of fraction deviation in the negative fractional deviation region. The detailed original algorithm was described in the previous report by Tan et al. [24]. For RNFL and GCC scanning map, images with an $\mathrm{SSI}<60$ needed to reacquired and finally the images with poor quality were excluded from the analysis.

\section{OCT angiography and retinal PVD measurements}

The OCT angiograms were acquired from a spectral domain OCT system (RTVue-XR Avanti, Optovue Inc., Fremont, CA), which has been described in previous study [25]. The OCT system combined with split-spectrum amplitude decorrelation angiography (SSADA) algorithm realized the visualization of retinal perfused vasculature. The OCT angiogram image scanning consisted a macular $(6.0 \times 6.0$ $\left.\mathrm{mm}^{2}\right)$ region and a optic disc $\left(4.5 \times 4.5 \mathrm{~mm}^{2}\right)$ region. The three dimension OCT angiograms was created through merging the two-dimensional scans and remove the motion artifacts with an orthogonal registration algorithm after scan acquisition. An en-face retinal angiograms were generated from the projection signal of flow from the internal limiting membrane (ILM) to the retinal pigment epithelium (RPE) automatically identified by The RTVue-XR Avanti software. The image quality of the OCT angiograms was determined by the signal strength index (SSI). Images with an SSI $\sim 60$ or less needed to be reacquired and finally the images with poor quality were excluded from the analysis. The eye-tracking function was used and it could improve the scanning image quality and decrease the residual motion artifacts to a large extent. The measurement of retinal PVD concentrated on the parafoveal region (an annulus consisted by an inner circle $(d=1 \mathrm{~mm})$ and an outer circle $(d=3$ $\mathrm{mm}$ ) ) and peripapillary region (a $700 \mu \mathrm{m}$-wide elliptical annulus extending outward from the optic disc boundary). The average and sectoral vessel density of these regions could be automatically quantified by the custom software (Fig. S1 E-H).

\section{Statistical analysis}

Intereye asymmetry for all parameters was defined as the absolute difference in the corresponding average or sectoral values between paired eyes. The intraeye asymmetry of all parameters was defined as the absolute difference between the values in the inferior and superior hemisphere of one eye. As both eyes of all patients were eligible for this study, one eye was randomly chosen for the analysis of intraeye asymmetry. Receiver operating characteristic (ROC) curve analysis was used to evaluate the diagnostic ability of the absolute intereye or intraeye difference to distinguish glaucomatous eyes from normal eyes. The AUROC values and the diagnostic sensitivities at both 80 and $95 \%$ specificity were obtained from this analysis. An AUROC of approximately 1.0 denotes perfect discrimination. The basic clinical characteristics difference between right and left eyes were calculated and their intereye asymmetry were compared between groups by independent sample $t$-test. Difference in sex distribution between groups was compared with a chi-square test. The above statistical analyses were performed with SPSS statistical software (version 20.0, SPSS, Inc., Chicago, IL). To decrease the experimental wise Type I error, the alpha values were adjusted by the following formula with Bonferroni multiple comparison adjustment method: $\alpha=0.05 /$ (number of parameters for comparison). For intereye asymmetric parameters analysis, the alpha value was reset at $0.0023(\alpha=0.05 / 22)$ and the values of $P<0.0023$ were considered to be significant. Similarly, for intraeye asymmetric parameters analysis, the alpha value was reset at $0.0125(\alpha=0.05 / 4)$ and the values of $P<0.0125$ were considered to be significant. According to the One-Sample Kolmogorov-Smirnov Test, all the continuous variables followed a normal distribution. The MedCalc statistical software (version 18.2.1, MedCalc, Inc., Ostend, Belgium) was used here to perform pairwise comparisons of ROCs [25], and $P<0.05$ were considered to be significant.

\section{Results}

\section{Basic information}

A total of 152 healthy subjects (male: female $=77: 75$ ) and 72 patients diagnosed with bilateral POAG (male: female $=$ 41:31) were enrolled in this study. The POAG patients included 40 early to moderate stage of POAG patients (male: female $=19: 21$ ). The mean ages of healthy subjects, total POAG patients and early to moderate stage of POAG patients were $44.61 \pm 11.75$ years, $45.90 \pm 13.25$ years and $49.05 \pm 14.41$ years, respectively. No significant difference in age or gender ratio was found between the healthy subjects and the total POAG or early to moderate stage of POAG patients(all $P<0.05)$. Comparisons of the intereye asymmetry of basic characteristics between healthy subjects and POAG patients are shown in Table 1 . The interocular differences of IOP and MD values in total 
Table 1 Comparison of intereye absolute differences in the ocular characteristic parameters among normal subjects, total and early to moderate stage of primary open-angle glaucoma patients

\begin{tabular}{lllllc}
\hline Parameters & Normal & Total POAG & $\begin{array}{l}\text { Early to moderate } \\
\text { stage of POAG }\end{array}$ & $P^{\mathrm{a}}$ & $P^{\mathrm{b}}$ \\
\hline$\triangle \mathrm{BCVA}$ & $0.08 \pm 0.13$ & $0.11 \pm 0.14$ & $0.09 \pm 0.12$ & 0.124 & 0.796 \\
$\triangle \mathrm{AL}(\mathrm{mm})$ & $0.18 \pm 0.22$ & $0.21 \pm 0.27$ & $0.24 \pm 0.32$ & 0.251 & 0.236 \\
$\triangle \mathrm{IOP}(\mathrm{mmHg})$ & $0.94 \pm 1.01$ & $1.79 \pm 2.31$ & $1.33 \pm 1.31$ & $0.004^{*}$ & 0.084 \\
$\triangle \mathrm{MD}(\mathrm{dB})$ & $0.50 \pm 0.78$ & $5.77 \pm 6.26$ & $2.74 \pm 2.48$ & $<0.001^{*}$ & $<0.001^{*}$
\end{tabular}

Data are presented as mean \pm standard deviation and compared with independent sample $t$-test

$B C V A$ best-corrected visual acuity, $A L$ axial length, $I O P$ intraocular pressure, $M D$ mean defect

$* P<0.05$

${ }^{\mathrm{a}}$ Normal vs. Total POAG group

${ }^{b}$ Normal vs. Early to moderate stage of POAG group
POAG patients were significantly greater than those in healthy subjects. For early to moderate stage of POAG patients, intereye asymmetry of MD was also significantly larger than that of healthy group.

\section{Intereye asymmetry of retinal PVD and neural structure parameters}

For both right and left eyes, the intereye peripapillary PVD asymmetry of total POAG patients was significantly higher than that of normal subjects (all $P<0.0023$ ). Several quadrants of the early to moderate stage of POAG group exhibited significantly larger interocular differences in peripapillary PVDs $(P<0.0023)$, with the exception of the superonasal sector $(P=0.077)$ and nasal sector $(P=0.021)$. Additionally, the intereye parafoveal PVD asymmetry of total POAG group has no significant difference compared to the healthy group (all $P>0.0023$ ). Besides, there is also no difference in the intereye asymmetry of parafoveal PVD between the healthy group and early to moderate stage of POAG group (all $P>0.0023$ ). However, significant differences in intereye structural asymmetry parameters (GCC and RNFL thickness) were observed between the healthy group and the total POAG (all $P<0.0023$ ) and early to moderate stage of POAG groups (all $P<0.0023$ ) (Table 2).

For each intereye asymmetry parameter, the sensitivity at a fixed specificity ( 80 or $95 \%$ ) and AUROC are listed in Table 3 and Fig. S2, and the pairwise comparisons of ROC curves are shown in Tables S1 and S2. The intereye asymmetry of the FLV\% showed the highest sensitivity (91.7 at $80 \%$ specificity and 87.5 at $95 \%$ specificity) and the largest AUROC value (0.944), followed by the intereye GLV\% asymmetry (0.944), intereye average GCC asymmetry (0.855), infer-hemi GCC asymmetry (0.845), superhemi GCC asymmetry (0.841), and average RNFL asymmetry (0.819). The AUROC values of the latter six parameters were not significantly different from each other (all $P>0.05$ ), whereas they were significantly superior to the infer-hemi RNFL, super-hemi RNFL, and average peripapillary PVD asymmetry (all $P<0.05$ ). However, the interocular differences in parafoveal PVDs had no diagnostic values in any subareas (all $P<0.0023$ ).

When distinguishing early to moderate stage of POAG patients from healthy subjects, the GLV and FLV\% were significantly superior to the other indices due to their excellent AUROC values (0.950 and 0.926, respectively) and sensitivity (95\% and $90 \%$ at $80 \%$ specificity; $90 \%$ and $87.5 \%$ at $95 \%$ specificity, respectively) (Table 3 and Fig. S3).

\section{Intraeye asymmetry of retinal PVD and neural structure parameters}

An independent sample $t$-test analysis showed significant differences in intraeye peripapillary PVD asymmetry, GCC thickness asymmetry, and RNFL thickness asymmetry for all comparison groups (healthy vs. total POAG, all $P<$ 0.0125; and healthy vs. early to moderate stage of POAG, all $P<0.0125)$. However, no significant difference was observed in intraeye parafoveal PVD asymmetry between the healthy and early to moderate stage of POAG groups $(P=0.821)$ (Table 4). Table 5 shows the diagnostic capabilities of intraeye asymmetry parameters, including peripapillary PVD, parafoveal PVD, GCC thickness, and RNFL thickness. The pairwise comparisons of ROC curves are listed in Tables S3 and S4. The intraeye GCC asymmetry $(\mathrm{AUROC}=0.772)$ and intraeye peripapillary PVD asymmetry $(A U R O C=0.768)$ showed modest diagnostic accuracy for detecting total POAG patients. The diagnostic abilities of these two parameters significantly outperformed intraeye RNFL asymmetry (AUROC $=0.667)(P<0.05)$.

Furthermore, for identifying the early to moderate stage of POAG patients, the intraeye GCC asymmetry (AUROC $=$ 0.790 ), intraeye peripapillary PVD asymmetry (AUROC $=$ $0.791)$, and intraeye RNFL asymmetry $($ AUROC $=0.691$ ) have fair and similar diagnostic values (all $P>0.05$ ). However, the intraeye parafoveal asymmetry had no diagnostic value for identifying the normal, total POAG 
Table 2 Comparison of intereye absolute differences in the retinal perfused vessel density and neural structure parameters among normal subjects, total and early to moderate stage of primary open-angle glaucoma patients

\begin{tabular}{|c|c|c|c|c|c|}
\hline Parameters & $\begin{array}{l}\text { Normal } \\
(n=152)\end{array}$ & $\begin{array}{l}\text { Total POAG } \\
(n=72)\end{array}$ & $\begin{array}{l}\text { Early to moderate } \\
\text { stage of POAG } \\
(n=40)\end{array}$ & $P^{\mathrm{a}}$ & $P^{\mathrm{b}}$ \\
\hline \multicolumn{6}{|c|}{ Peripapillary PVD (\%) } \\
\hline Average & $2.16 \pm 1.74$ & $6.35 \pm 5.18$ & $4.54 \pm 3.46$ & $<0.001 *$ & $<0.001 *$ \\
\hline Nasal & $3.34 \pm 2.81$ & $6.37 \pm 4.83$ & $4.58 \pm 3.60$ & $<0.001 *$ & 0.021 \\
\hline Temporal & $2.68 \pm 2.18$ & $7.81 \pm 7.10$ & $5.42 \pm 4.64$ & $<0.001^{*}$ & $<0.001 *$ \\
\hline Supernasal & $4.14 \pm 3.31$ & $6.72 \pm 6.15$ & $5.39 \pm 5.87$ & $<0.001^{*}$ & 0.077 \\
\hline Infernasal & $3.98 \pm 3.29$ & $8.34 \pm 6.98$ & $6.20 \pm 4.58$ & $<0.001^{*}$ & $0.001 *$ \\
\hline Supertempo & $4.26 \pm 3.11$ & $8.76 \pm 7.68$ & $6.59 \pm 5.16$ & $<0.001^{*}$ & $<0.001 *$ \\
\hline Infertempo & $3.87 \pm 3.11$ & $9.59 \pm 8.45$ & $9.05 \pm 3.48$ & $<0.001^{*}$ & $<0.001 *$ \\
\hline \multicolumn{6}{|c|}{ Parafoveal PVD (\%) } \\
\hline Average & $3.33 \pm 2.62$ & $4.49 \pm 3.85$ & $3.70 \pm 3.24$ & 0.013 & 0.462 \\
\hline Infer-hemi & $3.61 \pm 2.75$ & $4.77 \pm 4.29$ & $4.04 \pm 3.27$ & 0.015 & 0.398 \\
\hline Super-hemi & $3.47 \pm 2.70$ & $4.49 \pm 3.94$ & $3.82 \pm 3.64$ & 0.025 & 0.503 \\
\hline Nasal & $4.60 \pm 3.82$ & $5.64 \pm 4.61$ & $5.52 \pm 4.87$ & 0.076 & 0.205 \\
\hline Temporal & $2.68 \pm 3.11$ & $4.59 \pm 3.71$ & $5.42 \pm 4.48$ & 0.111 & 0.646 \\
\hline Inferior & $4.04 \pm 3.23$ & $5.56 \pm 4.27$ & $4.87 \pm 2.85$ & 0.003 & 0.136 \\
\hline Superior & $3.91 \pm 3.13$ & $5.39 \pm 4.38$ & $4.43 \pm 4.13$ & 0.004 & 0.385 \\
\hline \multicolumn{6}{|c|}{ GCC thickness $(\mu \mathrm{m})$} \\
\hline Average & $2.04 \pm 2.04$ & $10.92 \pm 11.05$ & $8.44 \pm 1.99$ & $<0.001^{*}$ & $<0.001 *$ \\
\hline Infer-hemi & $2.42 \pm 2.25$ & $12.64 \pm 15.16$ & $9.71 \pm 7.83$ & $<0.001^{*}$ & $<0.001 *$ \\
\hline Super-hemi & $2.24 \pm 2.13$ & $10.55 \pm 9.33$ & $8.63 \pm 7.33$ & $<0.001^{*}$ & $<0.001 *$ \\
\hline FLV\% & $0.26 \pm 0.55$ & $4.19 \pm 3.44$ & $4.04 \pm 3.34$ & $<0.001 *$ & $<0.001 *$ \\
\hline GLV\% & $0.50 \pm 0.76$ & $9.43 \pm 7.95$ & $7.83 \pm 5.62$ & $<0.001^{*}$ & $<0.001 *$ \\
\hline \multicolumn{6}{|c|}{ RNFL thickness $(\mu \mathrm{m})$} \\
\hline Average & $2.96 \pm 2.54$ & $11.44 \pm 10.13$ & $9.78 \pm 6.48$ & $<0.001 *$ & $<0.001 *$ \\
\hline Infer-hemi & $4.28 \pm 3.56$ & $11.96 \pm 10.68$ & $10.78 \pm 9.37$ & $<0.001 *$ & $<0.001 *$ \\
\hline Super-hemi & $3.78 \pm 2.83$ & $13.23 \pm 15.39$ & $11.65 \pm 16.17$ & $<0.001^{*}$ & $<0.001 *$ \\
\hline
\end{tabular}

Results are presented as the mean \pm standard deviation

$P V D$ perfused vessel density, Infer-hemi inferior hemisphere, Super-hemi superior hemisphere, GCC ganglion cell complex, $F L V \%$ focal loss volume percentage, GLV\% global loss volume percentage, $R N F L$ retinal nerve fiber layer

$* P<0.0023$, tested with independent sample $t$-test

${ }^{a}$ Normal vs. Total POAG group

${ }^{b}$ Normal vs. Early to moderate stage of POAG group
(AUROC $=0.497)$ or early to moderate stage of POAG groups $($ AUROC $=0.485)$ (Fig. S4).

\section{Discussion}

There is considerable clinical merit to using asymmetry values as diagnostic parameters, primarily because the comparison of indices between the fellow eyes or between the fellow hemispheres in a single eye excludes patientspecific factors, eye-specific factors, and the effect of systemic variables such as age, gender, AL, hypertension, diabetes, and cardiovascular disease to some extent.
However, the wide interindividual variation in the above factors has a larger impact on the results when the raw measurement parameters are compared between normal subjects and patients. Thus, in the current study, we evaluated the diagnostic capabilities of intereye and intraeye asymmetry in retinal neural structure and vascular parameters for detecting POAG.

In this study, the AUROC of interocular GCC thickness discriminated the total POAG patients $\quad$ (AUROC = 0.841-0.855), similar to the findings of prior studies that evaluated the diagnostic abilities of asymmetry maps of total or inner macular thickness $[9,11]$. Importantly, the diagnostic precision of intereye FLV and GLV\% asymmetry for 
Table 3 Diagnostic capabilities of intereye differences in the retinal perfused vessel density and structural parameters for differentiating primary openangle glaucoma from normal subjects

\begin{tabular}{|c|c|c|c|c|c|c|}
\hline \multirow[t]{3}{*}{ Parameters } & \multicolumn{6}{|c|}{ Comparison groups } \\
\hline & \multicolumn{3}{|c|}{ Normal-Total POAG } & \multicolumn{3}{|c|}{$\begin{array}{l}\text { Normal-Early to moderate stage } \\
\text { of POAG }\end{array}$} \\
\hline & ${ }^{\mathrm{a}} \mathrm{Sn}$ & ${ }^{\mathrm{b}} \mathrm{Sn}$ & AUROC (CI) & ${ }^{\mathrm{a}} \mathrm{Sn}$ & ${ }^{\mathrm{b}} \mathrm{Sn}$ & AUROC (CI) \\
\hline \multicolumn{7}{|c|}{ Peripapillary PVD (\%) } \\
\hline Average & 68.10 & 51.40 & $0.767 *(0.691-0.842)$ & 60.00 & 45.00 & $0.704 *(0.599-0.810)$ \\
\hline Nasal & 44.40 & 22.20 & $0.691 *(0.615-0.767)$ & 27.50 & 20.00 & $0.609(0.516-0.712)$ \\
\hline Temporal & 58.30 & 38.90 & $0.767 *(0.699-0.836)$ & 50.00 & 37.50 & $0.711^{*}(0.618-0705)$ \\
\hline Supernasal & 38.90 & 19.40 & $0.601(0.517-0.685)$ & 30.00 & 22.50 & $0.508(0.399-0.618)$ \\
\hline Infernasal & 54.20 & 29.20 & $0.704 *(0.627-0.781)$ & 42.50 & 25.00 & $0.642(0.538-0.746)$ \\
\hline Supertempo & 54.20 & 34.70 & $0.686^{*}(0.605-0.768)$ & 45.00 & 40.00 & $0.612(0.496-0.727)$ \\
\hline Infertempo & 54.20 & 43.10 & $0.694 *(0.612-0.777)$ & 47.50 & 42.50 & $0.643(0.528-0.758)$ \\
\hline \multicolumn{7}{|c|}{ Parafoveal PVD (\%) } \\
\hline Average & 26.40 & 16.70 & $0.564(0.481-0.646)$ & 17.50 & 15.00 & $0.514(0.413-0.616)$ \\
\hline Infer-hemi & 26.40 & 16.70 & $0.555(0.472-0.639)$ & 20.00 & 15.00 & $0.532(0.431-0.633)$ \\
\hline Super-hemi & 29.20 & 18.10 & $0.550(0.465-0.635)$ & 20.00 & 17.50 & $0.495(0.387-0.602)$ \\
\hline Nasal & 29.20 & 8.30 & $0.570(0.491-0.650)$ & 25.00 & 12.50 & $0.559(0.463-0.654)$ \\
\hline Temporal & 26.40 & 9.70 & $0.555(0.472-0.638)$ & 25.00 & 15.00 & $0.511(0.407-0.615)$ \\
\hline Inferior & 29.20 & 13.90 & $0.611(0.534-0.688)$ & 25.00 & 12.50 & $0.600(0.510-0.691)$ \\
\hline Superior & 37.50 & 18.10 & $0.592(0.510-0.674)$ & 25.00 & 17.50 & $0.511(0.407-0.614)$ \\
\hline \multicolumn{7}{|l|}{$\mathrm{GCC}(\mu \mathrm{m})$} \\
\hline Average & 80.60 & 61.10 & $0.855 *(0.793-0.916)$ & 85.00 & 75.00 & $0.880^{*}(0.814-0.946)$ \\
\hline Infer-hemi & 80.60 & 61.10 & $0.845 *(0.782-0.909)$ & 80.00 & 65.00 & $0.840 *(0.769-0.910)$ \\
\hline Super-hemi & 73.60 & 61.10 & $0.841 *(0.780-0.902)$ & 67.50 & 62.50 & $0.853^{*}(0.776-0.931)$ \\
\hline FLV\% & 91.70 & 87.50 & $0.944 *(0.907-0.982)$ & 90.00 & 87.50 & $0.926 *(0.864-0.987)$ \\
\hline GLV\% & 91.70 & 81.90 & $0.944 *(0.906-0.981)$ & 95.00 & 90.00 & $0.950 *(0.897-1.000)$ \\
\hline \multicolumn{7}{|l|}{ RNFL $(\mu \mathrm{m})$} \\
\hline Average & 66.70 & 51.40 & $0.819 *(0.755-0.884)$ & 67.50 & 62.50 & $0.829 *(0.746-0.912)$ \\
\hline Infer-hemi & 59.70 & 38.90 & $0.733 *(0.655-0.811)$ & 55.00 & 45.00 & $0.714 *(0.608-0.820)$ \\
\hline Super-hemi & 61.10 & 55.60 & $0.748 *(0.670-0.827)$ & 55.00 & 52.50 & $0.714 *(0.608-0.820)$ \\
\hline
\end{tabular}

$A U R O C$ areas under receiver operating characteristic curves, $\mathrm{Cl}$ confidence interval, Infer-hemi inferior hemisphere, Super-hemi superior hemisphere, GCC ganglion cell complex, FLV\% focal loss volume percentage, $G L V \%$ global loss volume percentage, $R N F L$ retinal nerve fiber layer

$* P<0.0023$, tested with receiver operating characteristic analysis

${ }^{a}$ Based on $80 \%$ specificity

${ }^{\mathbf{b}}$ Based on $95 \%$ specificity detecting total POAG and early to moderate stage of POAG patients were excellent and unsurpassed by any other index in our study and were not evaluated in other published reports.

The FLV and GLV\% are determined from the analysis of GCC loss patterns, including the fractional deviation map and the pattern deviation map. These two parameters were obtained from the calculation of the GCC loss volume with differing levels of focality, which has been shown to have good diagnostic accuracy in a previous study [24]. These pattern parameters had been shown to perform better than the raw RNFL and GCC thickness for identifying early-stage POAG eyes in which the presence of GCC loss is focal, but the global GCC thickness is within the normal range. In our study, the excellent diagnostic accuracy of intereye asymmetry for the FLV\% and GLV\% also reflected that they have the potential to aid in discriminating POAG.

Our study also found that compared to the intereye PVD asymmetry in similar regions, the neural structure parameters and relevant indices showed superior diagnostic ability for identifying POAG (Tables S3 and S4). The reasons for this result remain unknown, but the following explanations may account for the finding. In a previous study, Rao et al. [26] found that the average peripapillary PVD and average parafoveal PVD showed modest diagnostic ability but were significantly lower than those of the 
Table 4 Comparison intraeye absolute differences (between superior and inferior hemisphere) in the retinal perfused vessel density and structural parameters among normal subjects, total and early to moderate stage of POAG patients

\begin{tabular}{lllllc}
\hline Parameters & $\begin{array}{l}\text { Normal } \\
(n=152)\end{array}$ & $\begin{array}{l}\text { Total POAG } \\
(n=72)\end{array}$ & $\begin{array}{l}\text { Early to moderate } \\
\text { stage of POAG } \\
(n=40)\end{array}$ & $P^{\mathrm{a}}$ & $P^{\mathrm{b}}$ \\
\hline Peripapillary PVD $(\%)$ & $2.47 \pm 1.99$ & $6.66 \pm 5.83$ & $7.54 \pm 6.21$ & $<0.001^{*}$ & $<0.001^{*}$ \\
Parafoveal PVD $(\%)$ & $2.02 \pm 1.48$ & $2.08 \pm 1.77$ & $2.02 \pm 1.71$ & $<0.001 *$ & 0.821 \\
GCC thickness $(\mu \mathrm{m})$ & $2.50 \pm 2.51$ & $8.57 \pm 7.95$ & $9.66 \pm 8.77$ & $<0.001^{*}$ & $<0.001^{*}$ \\
RNFL thickness $(\mu \mathrm{m})$ & $6.37 \pm 4.42$ & $11.07 \pm 9.16$ & $12.45 \pm 10.82$ & $<0.001^{*}$ & $<0.001^{*}$ \\
\hline
\end{tabular}

Results are presented as the mean \pm standard deviation

$P V D$ perfused vessel density, $G C C$ ganglion cell complex, $R N F L$ retinal nerve fiber layer

${ }^{*} P<0.0125$, tested with independent sample $t$-test

${ }^{a}$ Normal vs. Total POAG group

${ }^{\mathrm{b}}$ Normal vs. Early to moderate stage of POAG group

Table 5 Diagnostic capabilities of intraeye asymmetry in retinal perfused vessel density and neural structure parameters for differentiating primary open-angle glaucoma from normal subjects

\begin{tabular}{|c|c|c|c|c|c|c|}
\hline \multirow[t]{3}{*}{ Parameters } & \multicolumn{6}{|c|}{ Comparison groups } \\
\hline & \multicolumn{3}{|c|}{ Normal-Total POAG } & \multicolumn{3}{|c|}{ Normal-Early to moderate stage of POAG } \\
\hline & ${ }^{\mathrm{a}} \mathrm{Sn}$ & ${ }^{\mathrm{b}} \mathrm{Sn}$ & $\operatorname{AUROC}(\mathrm{Cl})$ & ${ }^{\mathrm{a}} \mathrm{Sn}$ & ${ }^{\mathrm{b}} \mathrm{Sn}$ & $\operatorname{AUROC}(\mathrm{Cl})$ \\
\hline $\begin{array}{l}\text { Peripapillary } \\
\text { PVD }(\%)\end{array}$ & $59.70 \%$ & $59.70 \%$ & $0.768 *(0.699-0.837)$ & $63.60 \%$ & $40.00 \%$ & $0.791 *(0.715-0.866)$ \\
\hline Parafoveal PVD (\%) & $22.20 \%$ & $22.20 \%$ & $0.497(0.415-0.580)$ & $23.60 \%$ & $10.90 \%$ & $0.485(0.395-0.576)$ \\
\hline GCC thickness $(\mu \mathrm{m})$ & $66.70 \%$ & $66.70 \%$ & $0.772 *(0.696-0.847)$ & $69.10 \%$ & $50.90 \%$ & $0.790 *(0.707-0.874)$ \\
\hline RNFL thickness $(\mu \mathrm{m})$ & $38.90 \%$ & $38.90 \%$ & $0.667 *(0.590-0.745)$ & $41.80 \%$ & $36.40 \%$ & $0.691 *(0.606-0.776)$ \\
\hline
\end{tabular}

$A U R O C$ areas under receiver operating characteristic curves, $C l$ confidence interval, $P V D$ perfused vessel density, $G C C$ ganglion complex cell layer, $R N F L$ retinal nerve fiber layer

$* P<0.0125$, tested with receiver operating characteristic analysis

${ }^{\text {a }}$ Sn sensitivity based on $80 \%$ specificity

${ }^{\mathbf{b}} \mathrm{Sn}$ sensitivity based on $95 \%$ specificity

GCC thickness and RNFL thickness. The authors pointed out that the vasculature might not be the primary site in the pathogenesis of POAG, and the loss of retinal ganglion cells (RGCs) might be independent of retinal vascular factors. We considered that the differences in the measurement region size of different parameters might contribute to the significant disparity in their diagnostic abilities. The parafoveal PVD was only measured within an annulus centered at the fovea (an inner circle diameter $=1 \mathrm{~mm}$ and an outer circle diameter $=3 \mathrm{~mm}$ ). However, the image of the GCC thickness measurement map covered a $7 \times 7 \mathrm{~mm}$ circular region. Fig. S1 $(\mathrm{G}, \mathrm{H})$ clearly shows that the focal loss of the retinal microvascular network is visible in the superior, inferior and other areas in the total angiograms but not prominent in the measurement region. Similarly, the measurement region for the peripapillary PVD $(700 \mu \mathrm{m}$ elliptical annulus) was significantly smaller than that of the RNFL thickness map (3.45 mm circular annulus) (Fig. S1E, F). Therefore, the changes in retinal PVDs acquired might be underestimated, which lead to reduced diagnostic sensitivity and specificity.
In normal eyes, the highly symmetrical distribution of RGCs between the superior and inferior hemisphere of the retina contributed to the previous finding that the thicknesses of the RNFL and GCC were symmetrical between the upper and lower halves [27]. Prior studies have reported that intraeye asymmetry of the RNFL and macular thicknesses are useful parameters for discriminating POAG $[9,11,14]$. With regard to function, the GHT, which evaluates the hemifield asymmetry of visual sensitivity, has been proved to be a potential diagnostic parameter of earlystage glaucoma [15, 28-30]. Previous studies have reported that changes in PVD were closely correlated with retinal neural structure defects in both the macular and peripapillary regions [20, 21]. Thus, we hypothesized that PVD deficits might differ between the upper and lower hemispheres.

The results showed that the diagnostic capabilities of intraeye GCC and RNFL asymmetry were inferior to the results reported by other studies. The difference in methodology might contribute to the discrepancies among studies. Um et al. [13] developed the Macular Hemifield Test 
(MHT), which was derived from posterior pole asymmetry analysis and proved its high sensitivity and specificity for diagnosing early POAG. In Yamada's study [14], the logarithmic ratio of the upper to lower thickness of the ganglion cell layer was calculated as the new asymmetry parameter, and it had almost perfect discriminating ability regardless of glaucoma severity. However, our study used the absolute difference between the upper and lower halves as the asymmetric index. With a similar method, Sullivan et al. [9] chose the lower value (intraeye RNFL asymmetry values between 2 eyes) for the statistical analyses. We randomly selected one of two glaucomatous eyes to analyze the intraeye asymmetry to reduce the selection bias and enhance the credibility of the results.

The diagnostic ability of the intraeye peripapillary PVD asymmetry (AUROC $=0.768$ ) significantly outperformed that of intraeye parafoveal PVD asymmetry for identifying total POAG (AUROC $=0.497)$. The reason for this result remains unknown, but the following discussion may explain this finding. Table 4 shows that the intraeye asymmetry in the peripapillary region in POAG eyes was more obvious. This result, to some extent, indicated that a lesion in the vasculature in the peripapillary region might be more serious than a lesion in the parafoveal region. Previous studies observed that the response of the retinal vessels to increased stimulation (hyperoxia and acute increase in blood pressure) in the peripapillary region was significantly lower than in the parafoveal region $[25,31]$. The greater potential autoregulatory ability of the vasculature in the parafoveal region reflected its strong adaptability to environmental changes such that the risk of vascular lesions might be decreased to maintain normal blood supply and visual function. We hypothesized that due to this reason the difference in retinal PVD between the superior and inferior hemisphere in the parafoveal region was less than that in the peripapillary region.

There are several limitations in our study. First, we did not enroll suspected glaucoma patients, and therefore, we could not evaluate and compare the diagnostic accuracy of intereye and intraeye asymmetry parameters for discriminating preperimetric glaucomatous eyes. Moreover, the bilateral glaucoma patients enrolled in our study could not represent the overall glaucomatous population because there are still several unilateral glaucoma cases despite glaucoma is generally a bilateral disease. Furthermore, the small measurement region for retinal vessel density (parafoveal and peripapillary regions) might contribute to underestimation of the diagnostic power. Further development of software for OCT angiography will enlarge the retinal vessel density calculation area so that the ability to distinguish glaucoma patients from normal subjects might increase. Besides, our study used a simple method to analyze the intereye and intraeye asymmetry. With regard to intereye asymmetry, several subareas were examined when the major parameters were measured, and the differences were calculated in the corresponding subareas between the two eyes. However, intraeye asymmetry was only determined using the absolute difference between the upper and lower hemispheres in a single eye. Therefore, further studies should analyze the intraeye asymmetry values in detail. Furthermore, although anatomical structure and visual function always show obvious symmetric feature between the right and left eyes in healthy subjects, there still cases of asymmetricity involvement, it may have an influence on our results. In addition, for glaucomatous patients, we could not perform thorough examinations to eliminate the cardiovascular disease, which might affect the results to some extent.

Overall, according to the intereye asymmetry analysis, the neural structure indices were superior for screening glaucomatous eyes compared to microvascular indices in similar regions. Particularly, the intereye asymmetry of FLV\% and GLV\% were shown to be novel, excellent parameters for diagnosis of POAG patients. However, intraeye asymmetry parameters showed fair diagnostic ability for POAG patients.

\section{Summary}

\section{What was known before}

- The intereye differences in intraocular pressure (IOP), optic disc parameters, central corneal thickness, retinal nerve fiber layer (RNFL) thickness, macular thickness, and visual field defects were all associated with disease progression and the parameters had good diagnostic abilities for primary open-angle glaucoma.

\section{What this study adds}

- The intereye asymmetry of retinal microvascular parameters have modest diagnostic precision for detecting primary open-angle glaucoma, which are inferior to the neural structure parameters.

Acknowledgements All of authors would like to appreciate Jianfeng Luo, Fudan University, Shanghai, China, for assistance with the statistical analyses in this study.

Funding This research was supported by the Surface Project of National Natural Science Foundation of China (Grant No. 81770922, China), the project of Shanghai Municipal Commission of Health and Family Planning (Grant No. 201740204, China) the clinical science and technology innovation project of Shanghai Shenkang Hospital Development Center (SHDC12017X18), the western medicine guidance project of Shanghai Committee of Science and Technology 
(19411961600), the National Key Research \& Development Plan (2017YFC0108200) and the Shanghai Committee of Science and Technology (Grant No.13430710500, China).

\section{Compliance with ethical standards}

Conflict of interest The authors declare that they have no conflict of interest.

Publisher's note: Springer Nature remains neutral with regard to jurisdictional claims in published maps and institutional affiliations.

\section{References}

1. Weinreb RN, Khaw PT. Primary open-angle glaucoma. Lancet Lond Engl. 2004;363:1711-20. https://doi.org/10.1016/S01406736(04)16257-0

2. Poinoosawmy D. Frequency of asymmetric visual field defects in normal-tension and high-tension glaucoma. Ophthalmology. 1998;105:988-91. https://doi.org/10.1016/S0161-6420(98)96049-3

3. Williams AL, Gatla S, Leiby BE, Fahmy I, Biswas A, De Barros $\mathrm{DM}$, et al. The value of intraocular pressure asymmetry in diagnosing glaucoma. J Glaucoma. 2013;22:215-8. https://doi.org/10. 1097/IJG.0b013e318237bfb8

4. Miglior S, Torri V, Zeyen T, Pfeiffer N, Vaz JC, Adamsons I, et al. Intercurrent factors associated with the development of openangle glaucoma in the European glaucoma prevention study. Am J Ophthalmol. 2007;144:266-75. https://doi.org/10.1016/j.ajo.2007. 04.040

5. Li H, Healey PR, Tariq YM, Teber E, Mitchell P. Symmetry of optic nerve head parameters measured by the heidelberg retina tomograph 3 in healthy eyes: the Blue Mountains Eye study. Am J Ophthalmol. 2013;155:518-23.e1. https://doi.org/10.1016/j.ajo. 2012.09.019

6. Fansi AaK, Boisjoly H, Chagnon M, Harasymowycz P.J. Comparison of different methods of inter-eye asymmetry of rim area and disc area analysis. Eye Lond Engl. 2011;25:1590-7. https:// doi.org/10.1038/eye.2011.217

7. Iester M, Telani S, Frezzotti P, Vittone P, Traverso CE, Calabria $\mathrm{G}$, et al. Differences in central corneal thickness between the paired eyes and the severity of the glaucomatous damage. Eye Lond Engl. 2012;26:1424-30. https://doi.org/10.1038/eye.2012. 179

8. Sullivan-Mee M, Gentry JM, Qualls C. Relationship between asymmetric central corneal thickness and glaucomatous visual field loss within the same patient. Optom Vis Sci Publ Am Acad Optom. 2006;83:516-9. https://doi.org/10.1097/01.opx. 0000218433.49803.e7

9. Sullivan-Mee M, Ruegg CC, Pensyl D, Halverson K, Qualls C, et al. Diagnostic precision of retinal nerve fiber layer and macular thickness asymmetry parameters for identifying early primary open-angle glaucoma. Am J Ophthalmol. 2013;156:567-77.e1. https://doi.org/10.1016/j.ajo.2013.04.037

10. Mwanza J-C, Durbin MK, Budenz DL. Interocular symmetry in peripapillary retinal nerve fiber layer thickness measured with the cirrus HD-OCT in healthy eyes. Am J Ophthalmol. 2011;151:514-21.e1. https://doi.org/10.1016/j.ajo.2010.09.015

11. Lee S-Y, Lee EK, Park KH, Kim DM, Jeoung JW, et al. Asymmetry analysis of macular inner retinal layers for glaucoma diagnosis: Swept-source optical coherence tomography study. PLoS ONE. 2016;11:e0164866. https://doi.org/10.1371/journal. pone. 0164866

12. Levine RA, Demirel S, Fan J, Keltner JL, Johnson CA, Kass MA. Asymmetries and visual field summaries as predictors of glaucoma in the ocular hypertension treatment study. Invest Ophthalmol Vis Sci. 2006;47:3896-903. https://doi.org/10.1167/iovs.05-0469

13. Um TW, Sung KR, Wollstein G, Yun SC, Na JH, Schuman JS. Asymmetry in hemifield macular thickness as an early indicator of glaucomatous change. Invest Ophthalmol Vis Sci. 2012;53:1139-44. https://doi.org/10.1167/iovs.11-8373

14. Yamada H, Hangai M, Nakano N, Takayama K, Miyake M, et al. Asymmetry analysis of macular inner retinal layers for glaucoma diagnosis. Am J Ophthalmol. 2014;158:1318-29.e3. https://doi. org/10.1016/j.ajo.2014.08.040

15. Asman P, Heijl A. Evaluation of methods for automated Hemifield analysis in perimetry. Arch Ophthalmol Chic Ill 1960. 1992;110:820-6.

16. Asman P, Heijl A. Glaucoma Hemifield test. Automated visual field evaluation. Arch Ophthalmol Chic Ill 1960. 1992;110: 812-9.

17. Jia Y, Morrison JC, Tokayer J, Tan O, Lombardi L, Baumann B, et al. Quantitative OCT angiography of optic nerve head blood flow. Biomed Opt Express. 2012;3:3127-37. https://doi.org/10. 1364/BOE.3.003127

18. Huang D, Jia Y, Gao SS, Lumbroso B, Rispoli M, et al. Optical coherence tomography angiography using the optovue device. Dev Ophthalmol. 2016;56:6-12. https://doi.org/10.1159/ 000442770

19. Liu L, Jia Y, Takusagawa HL, Pechauer AD, Edmunds B, Lombardi $\mathrm{L}$, et al. Optical coherence tomography angiography of the peripapillary retina in glaucoma. JAMA Ophthalmol. 2015;133:1045-52. https://doi.org/10.1001/jamaophthalmol.2015.2225

20. Wang $X$, Jiang $C$, Ko T, Kong XM, Yu XB, Wang M, et al. Correlation between optic disc perfusion and glaucomatous severity in patients with open-angle glaucoma: an optical coherence tomography angiography study. Graefes Arch Clin Exp Ophthalmol Albrecht Von Graefes Arch Für Klin Exp Ophthalmol. 2015;253:1557-64. https://doi.org/10.1007/s00417-0153095-y

21. Xu H, Yu J, Kong X, Sun XH, Jiang CH. Macular microvasculature alterations in patients with primary open-angle glaucoma: A cross-sectional study. Med (Baltim). 2016;95:e4341. https://doi.org/10.1097/MD.0000000000004341

22. Rao HL, Pradhan ZS, Weinreb RN, Riyazuddin M, Dasari S, Puttaiah NP, et al. A comparison of the diagnostic ability of vessel density and structural measurements of optical coherence tomography in primary open angle glaucoma. PLoS ONE. 2017;12: e0173930. https://doi.org/10.1371/journal.pone.0173930

23. Rao HL, Kadambi SV, Weinreb RN, Puttaiah NK, Pradhan ZS, Rao DAS, et al. Diagnostic ability of peripapillary vessel density measurements of optical coherence tomography angiography in primary open-angle and angle-closure glaucoma. $\mathrm{Br} \mathrm{J}$ Ophthalmol. 2017;101:1066-70. https://doi.org/10.1136/bjophthalmol2016-309377

24. Tan O, Chopra V, Lu AT-H, Schuman JS, Ishikawa H, Wollstein $\mathrm{G}$, et al. Detection of macular ganglion cell loss in glaucoma by Fourier-domain optical coherence tomography. Ophthalmology. 2009;116:2305-14. https://doi.org/10.1016/j.ophtha.2009.05.025.

25. Xu H, Deng G, Jiang C, Kong XM, Yu J, Sun XH, et al. Microcirculatory responses to hyperoxia in macular and peripapillary regions. Invest Ophthalmol Vis Sci. 2016;57:4464-8. https://doi.org/10.1167/iovs.16-19603

26. Rao HL, Pradhan ZS, Weinreb RN, Reddy HB, Riyazuddin M, Dasari S, et al. Regional comparisons of optical coherence tomography angiography vessel density in primary open-angle glaucoma. Am J Ophthalmol. 2016;171:75-83. https://doi.org/10. 1016/j.ajo.2016.08.030

27. Nakano N, Hangai M, Nakanishi H, Mori S, Nukada M, Kotera $\mathrm{Y}$, et al. Macular ganglion cell layer imaging in preperimetric glaucoma with speckle noise-reduced spectral domain optical 
coherence tomography. Ophthalmology. 2011;118:2414-26. https://doi.org/10.1016/j.ophtha.2011.06.015

28. Susanna R, Nicolela MT, Soriano DS, Carvalho C, et al. Automated perimetry: a study of the glaucoma hemifield test for the detection of early glaucomatous visual field loss. J Glaucoma. 1994;3:12-6.

29. Duggan C, Sommer A, Auer C, Burkhard K, et al. Automated differential threshold perimetry for detecting glaucomatous visual field loss. Am J Ophthalmol. 1985;100:420-3.
30. Sommer A, Enger C, Witt K. Screening for glaucomatous visual field loss with automated threshold perimetry. Am J Ophthalmol. 1987; 103:681-4.

31. Zong $\mathrm{Y}, \mathrm{Xu} \mathrm{H}, \mathrm{Yu}$ J, Jiang $\mathrm{CH}$, Kong XM, He Y, et al. Retinal vascular autoregulation during phase IV of the valsalva maneuver: An optical coherence tomography angiography study in healthy chinese adults. Front Physiol. 2017;8. https://doi.org/10.3389/ fphys.2017.00553 